\title{
Does progressive resistance and balance exercise reduce falls in residential aged care? Randomized controlled trial protocol for the SUNBEAM program
}

\author{
This article was published in the following Dove Press journal: \\ Clinical Interventions in Aging \\ 21 February 2014 \\ Number of times this article has been viewed
}

\section{Jennifer Hewitt ${ }^{\prime}$ \\ Kathryn M Refshauge' \\ Stephen Goodall ${ }^{2}$ \\ Timothy Henwood ${ }^{3}$ \\ Lindy Clemson'}

'Faculty of Health Sciences, University of Sydney, ${ }^{2}$ Centre for Health

Economic Research and Evaluation, University of Technology, Sydney, NSW, ${ }^{3}$ University of Queensland/Blue Care Research and Practice Development Centre, The University of Queensland, Brisbane, QLD, Australia
Correspondence: Jennifer Hewitt Physiotherapy, Faculty of Health Sciences The University of Sydney, Arthritis and Musculoskeletal Research Laboratory, 75 East Street, Lidcombe, NSW 22/4, Australia

Tel +6I 427226045

Fax +61755905117

Email jhew4562@uni.sydney.edu.au

\begin{abstract}
Introduction: Falls are common among older adults. It is reported that approximately $60 \%$ of residents of aged care facilities fall each year. This is a major cause of morbidity and mortality, and a significant burden for health care providers and the health system. Among community dwelling older adults, exercise appears to be an effective countermeasure, but data are limited and inconsistent among studies in residents of aged care communities. This trial has been designed to evaluate whether the SUNBEAM program (Strength and Balance Exercise in Aged Care) reduces falls in residents of aged care facilities.
\end{abstract}

Research question: Is the program more effective and cost-effective than usual care for the prevention of falls?

Design: Single-blinded, two group, cluster randomized trial.

Participants and setting: 300 residents, living in 20 aged care facilities.

Intervention: Progressive resistance and balance training under the guidance of a physiotherapist for 6 months, then facility-guided maintenance training for 6 months.

Control: Usual care.

Measurements: Number of falls, number of fallers, quality of life, mobility, balance, fear of falling, cognitive well-being, resource use, and cost-effectiveness. Measurements will be taken at baseline, 6 months, and 12 months.

Analysis: The number of falls will be analyzed using a Poisson mixed model. A logistic mixed model will be used to analyze the number of residents who fall during the study period. Intentionto-treat analysis will be used.

Discussion: This study addresses a significant shortcoming in aged care research, and has potential to impact upon a substantial health care problem. Outcomes will be used to inform care providers, and guide health care policies.

Keywords: balance, strength, training, falls, nursing care, cost-effectiveness

\section{Introduction}

The size of the population aged 75 years and older is projected to grow to more than double in the next 20 years. ${ }^{1}$ The number of people living in residential aged care, the number of fall-related hospital admissions, and the costs of follow-up care are also expected to rise. ${ }^{2}$ Identification and implementation of effective interventions to reduce falls in this setting has the potential to significantly benefit older individuals, and to reduce the health care burden.

To date, the majority of studies have focused on falls among community-dwelling older adults. However, the number of falls among residents of aged care facilities is 
reported to be three times greater. ${ }^{3}$ The consequences of falls are often traumatic, and include reduced independence, injury, and death. ${ }^{3,4}$ The burden upon society is also substantial. Australian data show that, while residents of aged care facilities form only one-eighteenth of the older population, the cost of their falls is greater than one-fifth of the total cost of falls, to the health system. ${ }^{5}$ Therefore, a recent economic evaluation of projected costs of health care recommended urgent action, to prevent falls in aged care facilities., ${ }^{2,6}$

Falls are not considered to be purely random events, but can be predicted to arise from a number of risk factors, including: visual impairment, vitamin D deficiency, foot pain, incontinence (particularly urgency), poor nutrition, psychoactive medications, cardiac arrhythmia, reduced lower limb muscle strength, and impaired balance and gait. ${ }^{7-10}$ There have been many randomized controlled trials, Cochrane Collaboration reviews, and other systematic reviews conducted, to explore the effectiveness of a range of fall prevention strategies, including single interventions (targeting one risk factor), multiple interventions (targeting more than one risk factor), and multifactorial interventions (individualizing the interventions to the participant's risk factors). ${ }^{3}$ There is evidence that exercise, as a single intervention, can prevent falls in older community dwellers. ${ }^{911-15}$ A recent review from the Cochrane Collaboration that examined fall prevention interventions in residential aged care facilities (RACFs) and hospitals identified eleven studies which had tested exercise as a single intervention. The pooled results returned inconsistent data; it was concluded that carefully-designed research into supervised exercise for falls prevention in this setting is essential. ${ }^{3}$ Clinical practice guidelines currently recommend the following key components for exercise programs: high-challenge balance training, moderateto-high intensity progressive resistance training (PRT), and a total of at least 50 hours of exercise. ${ }^{12}$

Therefore, this study aims to test whether the SUNBEAM program (Strength and Balance Exercise for Aged care), which is based on key components of successful communitybased programs, will reduce falls in the high-risk group of residents of aged care facilities.

The key research questions are:

- Is a supervised, group-based, PRT and balance exercise program more effective than usual care for the prevention of falls among residents, during a 12-month follow-up period?

- Does the program result in improvements in secondary outcomes: quality of life, cognition, mobility, and confidence?

- Is the program cost-effective?

\section{Method}

\section{Design}

This will be an assessor-blinded, two-group, clusterrandomized, controlled trial. It will be funded by a donation from Domain Principal Group (Sydney, NSW, Australia), and in-kind support from HUR Health and Fitness Equipment (Birkdale, QLD, Australia). Concealed allocation and intention-to-treat analysis will be used. Measurements will be taken at baseline, immediately following the intervention (6 months), and at 12 months after randomization, to examine the maintenance of any intervention effects. The study protocol has been approved by The University of Sydney Ethics Committee (Approval number 14995), and has been registered in the Australia and New Zealand Clinical Trials Registry (Registration number: ACTRN12613000179730). The trial's results will be reported using domains and categories described in the taxonomy developed by the Prevention of Falls Network Europe, to allow future synthesis of evidence, or study replication. ${ }^{16} \mathrm{~A}$ flowchart of the trial design is provided in Figure 1.

\section{Residential aged care facilities and participants}

We will recruit 300 residents and 20 RACFs from northern New South Wales and South East Queensland, Australia. The inclusion criteria for RACFs are: 1) to have a mix of high-care residents ("nursing home" residents, who require daily care by - or under the supervision of - registered nurses) and/or low-care residents ("hostel" residents, who need some assistance, but do not have complex health care needs); 2) to be likely to have 15 residents willing to participate; and 3) the facility manager consents to participation in the trial and to the allocation of staff time. Staff will assist with generating a list of potential participants (using the trial's inclusion/ exclusion criteria), approaching potential participants, to invite them to volunteer for the trial, and (where relevant) will assist with supervision of the exercise sessions over the 12-month trial period. Enduring power of attorney holders, where present, will be contacted by mail, for each potential participant.

Participants will include men and women who permanently reside in residential aged care and are able to understand English to a level where they can comprehend the participant information statement, complete the consent form, and carry out self-report outcome measures without an interpreter. Residents with a terminal or unstable illness, significant advanced cognitive decline (Mini Mental State Examination $^{17} \leq 15$ ), or physical symptoms that preclude 
Residential aged care facility managers volunteer their center for participation in the trial $(n=20)$

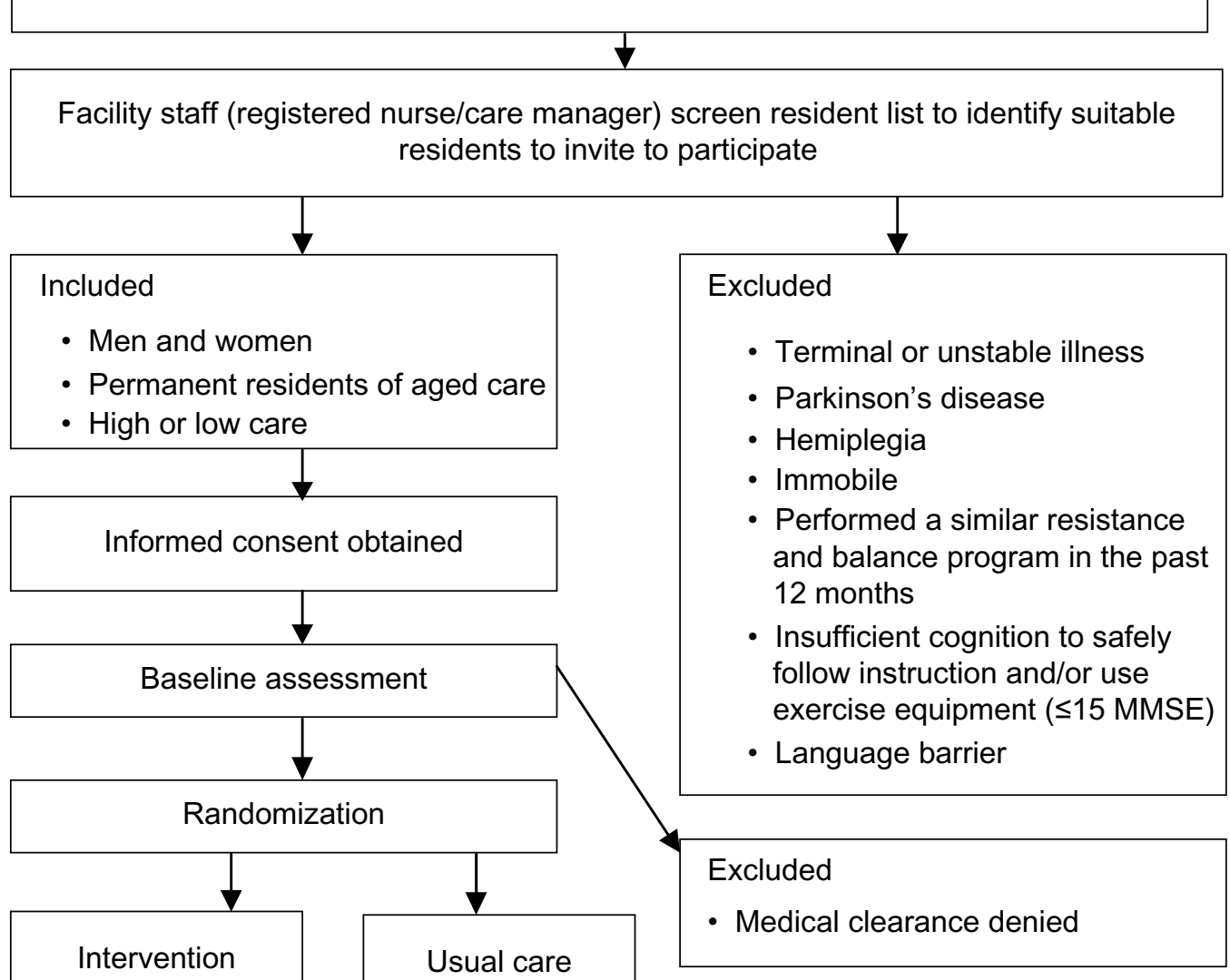

Figure I Flowchart of study design.

Abbreviation: MMSE, Mini Mental State Examination. 
the safe use of exercise equipment in a group setting (eg, Parkinson's Disease or hemiplegia) will be ineligible. Other criteria will exclude those who are permanently wheelchair- or bed-bound, and those who have performed a similar balance and/or resistance training program within the previous 12 months.

\section{Outcome measures}

Data will be collected at baseline, 6 months, and 12 months by blinded assessors. Baseline measurements will be taken as soon as possible after recruitment, and before randomization. In addition to falls data, a range of demographic information and known risk factors for falls will be recorded at each assessment, including comorbid conditions, medications, environmental hazards, use of a mobility aid, and incontinence. ${ }^{7,9,18}$

\section{Primary outcome}

The primary outcomes will be the proportion of residents who fall (fallers), and the number of falls for each participant (fall rate), during the 12-month trial period. The definition of a fall will be: "an unexpected event in which the participant comes to rest on the ground, floor, or lower level", as derived by consensus statement ${ }^{19}$ and used in recent reviews by the Cochrane Collaboration. ${ }^{9}$ Individual falls will be recorded every month for the 12-month study period, by auditing aged care facility incident reports. ${ }^{20-22}$ In addition, at the time of each assessment, participants will be asked directly if they have fallen. It is acknowledged that it would be preferable to incorporate multiple approaches to collecting falls data, to improve accuracy. However, this is beyond the resources available to this study.

\section{Secondary outcomes}

Quality of life (QOL): The aim will be to describe what aspects of QOL are affected, and to what extent, if any, QOL is improved in the Intervention group. QOL will be measured using the 36- item short form health survey (SF-36) and the EuroQol -5 dimension - 5 level (EQ-5D-5L) instruments. The SF-36 is the most widely used measure of general health. ${ }^{23}$ EQ-5D-5L is a 5-level version of the widely used EQ-5D scale. EQ-5D-5L is cognitively undemanding and takes only a few minutes to complete; it is potentially ideal for the RACF population. ${ }^{24}$ We will test the validity and acceptability of using the EQ-5D-5L, relative to the SF-36, in the RACF setting.

Measures of balance and gait will be taken, as these have been identified as potential risk factors ${ }^{9}$ for falls, that may be remediable with exercise. ${ }^{7,12}$ The Short Physical Performance
Battery (SPPB) ${ }^{25}$ will be used to evaluate balance, gait, strength, and endurance. The Step Test ${ }^{26}$ will be used to assess dynamic balance and agility.

The University of Alabama, Birmingham Life-Space Assessment will be used to assess extent of mobility and frequency of movement. ${ }^{27,28}$ The scores represent how much an individual actually mobilizes over a 4-week period (rather than the distance that they are capable of).

Fear of falling will be measured using the Falls Efficacy Scale International (FES-I), ${ }^{29}$ which evaluates confidence in avoiding falls when performing basic activities of daily living, and has been shown to maintain good measurement properties in persons with or without moderate cognitive impairment and, when administered in an interview format, in frail older persons. ${ }^{30}$

Cognition will also be assessed, as cognitive impairment has been identified as a risk factor for falls. ${ }^{31}$ A recent systematic review and meta-analysis of the effects of exercise training on older people with cognitive impairment and dementia found that training resulted in improvements in health-related physical fitness and cognitive function. ${ }^{32}$ Cognitive status will be measured using the Addenbrooke's Cognitive Examination Revised (ACE-R), a brief cognitive test that assesses five cognitive domains: attention, memory, verbal fluency, language, and visuospatial abilities. ${ }^{33}$

\section{Intervention (SUNBEAM program)}

Participants allocated to the Intervention group will perform an exercise program, conducted in group settings (of approximately 10 participants) in two stages during the 12-month trial period. The first stage ( $0-6$ months) will comprise of progressive resistance (strength) training, with static and dynamic standing balance exercises. ${ }^{7,11,34,35}$ The exercises will be prescribed, and supervised by an exercise professional, such as a physiotherapist or an exercise physiologist. An RACF staff member (diversional therapist or physiotherapy assistant) will cosupervise during this period, to help maintain safety. If there are several participants in an exercise group who demonstrate a need for close supervision (eg, lower cognitive functioning; very poor dynamic balance), an extra supervisor will be recruited.

The second stage (7-12 months) will consist of a maintenance program of resistance, weight-bearing balance, and functional exercises. ${ }^{12}$ Each group will continue to be supervised by the RACF staff member who worked with the group during the initial intervention stage. Exercise doses for this stage will be prescribed by the exercise professional on completion of the initial training stage. 


\section{Stage I (0-6 months): PRT and balance exercise}

PRT will target large muscle groups in the lower and upper limbs and trunk, using specialized pneumatic resistance equipment (HUR Australia Pty Ltd, Birkdale, QLD, Australia). Specifically, the resistance devices to be used include: knee extension, knee flexion (leg curl), abdominal curl, back extension, hip abduction, hip adduction, elbow and shoulder extension (dip), and leg press. Dosages will be individually prescribed, so as to enable each participant to achieve 2-3 sets of 10-15 repetitions of each exercise. ${ }^{7,36}$ Dosages will be gradually adjusted as participants' abilities change throughout the course of the program. ${ }^{12}$ If an individual has a specific comorbid condition that precludes them from safely using an item of equipment, a substitute exercise will be prescribed, using resistance bands to target the same muscle groups. Elbow and shoulder flexion exercises will be performed using resistance bands.

Balance exercises will include a combination of heel and toe raises, stepping in different directions, single leg standing, step-ups, and task-specific balance work (eg, reaching outward from the base of support while standing, sitting, and standing and turning). Balance exercises will be upgraded by: 1) reducing hand support and/or 2) narrowing the base of support, and/or 3) introducing a cognitive challenge (eg, counting backwards while performing exercise) or performing exercise with the eyes closed. ${ }^{7,12}$

Sessions will be of 1-hour duration, and will be conducted twice per week over a 6-month period. ${ }^{7,12}$ After 6 months, the resistance training equipment will be moved to the nextincluded RACF of the Intervention group.

No structured or standardized sessions of education for falls prevention will be conducted during Stage 1. However, if a participant shows unsafe behavior during sessions (eg, wearing unsafe footwear; attempting to walk without mobility aid), the supervisory exercise professional will provide specific feedback that is consistent with standard practice and their duty of care.

\section{Stage 2 (7-12 months): Maintenance exercise}

A maintenance program that includes resistance exercise (using bands), balance, weight-bearing, and functional exercises will complete the remaining 6 months of the study. Participants will be asked to sign their names in a book at each visit, to record attendance. Sessions will be supervised by the RACF staff member (diversional therapist or physiotherapy assistant) who was involved in cosupervising the initial training sessions (Stage 1) and a volunteer, if deemed necessary by the exercise professional. The exercise program will include: 1) performance of the standing and sitting balance exercise at the level safely achieved by the end of the initial 6-month training, using a setup designed to optimize safety; 2) sit-to-stand exercises; and 3) resistance band exercises for the trunk and upper limbs (sitting or standing). Classes will be conducted twice per week for 30 minutes per session.

The total number of exercise sessions attended in both Stage 1 and Stage 2, as well as the exercises performed at each session by each participant, will be recorded. Acceptability will be determined by participant adherence to the program; information about acceptability will be determined by exit interviews.

\section{Control group}

Participants who are allocated to the control group will continue with usual care, without the introduction of the SUNBEAM program. Usual care may include activities, games, and hobbies, which will be recorded at each assessment.

\section{Procedure}

A list of all RACFs within northern New South Wales and South East Queensland was generated in 2012, by Internet searching and using local telephone directories. A letter was sent to each facility to invite expressions of interest. In addition, presentations were given by a research team member at relevant industry forums, to explain the trial objectives and protocol, and to answer any questions.

Staff at participating RACFs will use the inclusion and exclusion criteria to generate a list of eligible residents. All eligible residents will be informed by RACF staff that participants are being recruited for a long-term exercise study, to take place across multiple sites, and will be provided with a participant information sheet. Residents who consent to volunteer for the trial will be contacted by the research team, to organize final screening and a baseline assessment. Letters will be sent to potential participants' medical practitioners, to seek medical clearance. Each participant's next of kin (or enduring power of attorney) will also be advised, by mail, of the participant's consent to join the trial. After baseline measures are completed, the RACFs will be randomized (to receive either the SUNBEAM Program or usual care) by a researcher, independent of baseline assessment, using a computer-generated randomization schedule.

Falls will be recorded monthly for the duration of the trial. All other outcome measures will be taken at 6 months 
(ie, immediately post-intervention) and at 12 months, by assessors blinded to group allocation.

Residents of the first included RACF were recruited in August 2012. It is anticipated that recruitment will continue over the next 4 years.

\section{Data analysis}

\section{Effectiveness of intervention}

The primary analysis will be based on an intention-to-treat approach. This will be compared to per-protocol analyses. Summary outcomes will be presented at the cluster level, using standard measures. Analyses will also be conducted at the participant level, but adjusted appropriately for clustering of participants within RACFs, using mixed models. A logistic mixed model will be used to analyze the number of residents who fall during the study period (binary outcome). The number of falls (a count outcome) will be analyzed using a Poisson mixed model. To adjust for loss of follow-up, which may be significant in this cohort, a multilevel survival analysis will be conducted, with the outcome being time to first fall (and first fracture). All regression models will include the treatment group as an explanatory variable, and also a random effect for RACFs, to adjust for any clustering effects. Baseline characteristics will be compared between the two groups; any potential confounding factors that are found not to be balanced among groups, such as age, will be included as covariates in the regression models. Model assumptions will be tested, and appropriate adjustment to the analysis, such as logarithmic transformation of skewed variables, will be made as necessary.

Pre-specified subgroup analyses will be performed on the following variables: 1) level of care; 2) previous faller; 3) number of falls in the 12 months prior to inclusion, 4) program adherence, and dosage of exercise completed; 5) age; and 6) presence of other known risk factors for falls. Interactions between falls and ability to mobilize, ${ }^{37}$ physical performance measures, ${ }^{8}$ fear of falling, and QOL will also be examined.

\section{Cost-effectiveness analysis}

A recent Cochrane Collaboration review has identified a need for economic evaluation of falls prevention interventions. ${ }^{34}$ A stepped cost benefit analysis will be undertaken, to examine the costs of providing the exercise program, and any cost offsets due to reduced health services use resulting from fall incidents. Program costs will include the capital cost of exercise equipment, the cost of any additional training material, and the costs of the exercise professional and supervisory staff. Health service use during the 12-month trial period will be determined from monthly auditing of RACF records, to extract data specific to fall incidents; these will include: 1) any medical services utilized, such as medical practitioner visits; 2) transfers to hospital; 3) hospital admissions; 4) number of nights admitted; 5) procedures performed; 6) follow-up visits; 7) rehabilitation; and 8) pharmaceutical drug usage. The total health service costs will be derived by multiplying the units of resource used by the relevant factor: the Australian Government's Medicare Schedule Benefit item fee, Pharmaceutical Benefits Scheme price, or the Australian Refined Diagnosis-Related Group cost. ${ }^{38,39,40}$

An incremental cost-effectiveness ratio will also be calculated, relative to the control group, as cost per quality of life year gained. To perform this analysis, the health benefits associated with the program will be estimated using the SF-36. A preference-based single utility measure, using Australian preference weights, will be derived from SF-36 using the Short Form-6 Dimensions (SF-6D) as described by Norman et al. ${ }^{41} \mathrm{~A}$ supplementary analysis using the EQ5D-5L will also be conducted. ${ }^{42} \mathrm{~A}$ within-trial time horizon will form the base case analysis. Extrapolations beyond the trial period (eg, a 5-year time horizon) will be based on various assumptions about the sustainability of the treatment effect. Sensitivity analyses will be undertaken, to explore the robustness and validity of the cost-effectiveness data, and to test any assumptions used in the economic model.

\section{Sample size}

The study has been powered with respect to the primary outcome: falls. It is estimated that approximately $60 \%$ of participants in the usual care group will sustain at least one fall during the 12 months of follow-up. ${ }^{8}$ Meta-analysis of pooled community and residential aged care studies of incorporating high-dose exercise ( $>50$ hours) and high level balance training have demonstrated a reduction in fall rates of $38 \% .{ }^{12}$ The intervention in this study will contain these components, but will be specific to residents of RACFs. Assuming that participation reduces the proportion of falls and fallers, the exercise program will be considered successful if, at 12 months after randomization, only $40 \%$ of the intervention group have fallen - an absolute difference of $20 \%$.

Twenty RACFs (clusters) will be recruited, with outcomes to be collected for an average of 15 residents per facility. Several studies of RACFs ${ }^{11,43}$ indicate that the intracluster correlation coefficient (ICC) is close to zero $(<0.01)$. ICCs for clinical and physical activity variables ranged between $0-0.08$ in three cluster trials of residential health care. ${ }^{43}$ With a zero ICC, we 
would need to recruit 194 residents of RACFs, in order to detect a $20 \%$ difference, with $80 \%$ power, at a $5 \%$ two-sided significance level. We will recruit 300 residents, to allow us to detect a $20 \%$ absolute difference, with $80 \%$ power, if ICC $=0.01$, allowing for a conservative $25 \%$ dropout (given the participants' ages and the presence of comorbid conditions).

\section{Discussion}

Despite a plethora of research into the area of falls prevention, there is little conclusive evidence available to show effective ways of reducing falls in adults in residential care. This trial utilizes an intervention that has been proven to be effective in community-dwelling older people, and tests whether these results can be extrapolated to the residential care setting within a more supervised and supported environment. If the intervention is shown to be effective, there is potential for this study to have both immediate and long-term impact, in terms of benefits to older individuals, and decreased direct health care costs.

For older people living in RACFs, potential direct benefits of this exercise program are reduced probabilities of falling and the sequelae of falls, such as increased mortality, morbidity, injury, hospitalization, and loss of confidence, along with reduced mobility and reduced quality of life.

For the health care system, fewer fall-related hospital admissions will reduce costs to society, and help to improve access to hospitals. Benefits for health departments will be realized if the exercise program is cost-effective, and if the program is accepted by RACFs and their residents.

Finally, the intervention is simple for RACFs: it can be rolled out easily, to have far-reaching impact. Its implications may include reducing the health care burden of falls, improving the well-being of residents of RACFs, and contributing to the health policy debate, by challenging current residential aged care funding models.

\section{Acknowledgments}

The authors gratefully acknowledge Domain Principal Group for donating to support the running costs of this trial. We also thank HUR Australia Pty Ltd for providing the resistance training equipment used in this trial, and Christopher Turner of Allied Connect Pty Ltd., Varsity Lakes, QLD, Australia, for contributing to the project's conception and initial design, and for assistance with recruiting facilities to participate in the trial.

\section{Disclosure}

None of the authors has any actual or potential conflict of interest to disclose, including any financial, personal, or other relationships with other people or organizations that could inappropriately influence this work.

\section{References}

1. Australian Bureau of Statistics. 3236.0 - Household and Family Projections, Australia, 2006 to 2031 [Internet]. 2010; cited February 14, 2013. Available from: http://www.abs.gov.au/ausstats/abs@.nsf/ $\mathrm{mf} / 3236.0$ Accessed November 17, 2013.

2. Church J, Goodall S, Norman R, Haas M. An economic evaluation of community and residential aged care falls prevention strategies in NSW. N S W Public Health Bull. 2011;22(3-4):60-68.

3. Cameron ID, Gillespie LD, Robertson MC, et al. Interventions for preventing falls in older people in care facilities and hospitals. Cochrane Database Syst Rev. 2012;12:CD005465.

4. Fatovich DM, Jacobs IG, Langford SA, Phillips M. The effect of age, severity, and mechanism of injury on risk of death from major trauma in Western Australia. J Trauma Acute Care Surg. 2013;74(2):647-651.

5. Watson W, Clapperton A, Mitchell R. The burden of fall-related injury among older persons in New South Wales. Aust N Z J Public Health. 2011;35(2):170-175.

6. Church J. Goodall S, Norman R, Haas M R. The cost-effectiveness of falls prevention interventions for older community-dwelling Australians. Australian and New Zealand Journal of Public Health. 2012;36(3):241-248.

7. Tiedemann A, Sherrington C, Close JC, Lord SR. Exercise and Sports Science Australia position statement on exercise and falls prevention in older people. J Sci Med Sport. 2011;14(6):489-495.

8. Lord SR, March LM, Cameron ID, et al. Differing risk factors for falls in nursing home and intermediate-care residents who can and cannot stand unaided. J Am Geriatr Soc. 2003;51(11):1645-1650.

9. Gillespie L, Handoll H. Prevention of falls and fall-related injuries in older people. Inj Prev. 2009;15(5):354-355.

10. Lord SR. Aging and falls: causes and prevention. $J$ Musculoskelet Neuronal interact. 2007;7(4):347.

11. Sherrington C, Whitney JC, Lord SR, Herbert RD, Cumming RG, Close JC. Effective exercise for the prevention of falls: a systematic review and meta-analysis. J Am Geriatr Soc. 2008;56(12):2234-2243.

12. Sherrington C, Tiedemann A, Fairhall N, Close JC, Lord SR. Exercise to prevent falls in older adults: an updated meta-analysis and best practice recommendations. N SW Public Health Bull. 2011;22(3-4):78-83.

13. Panel on Prevention of Falls in Older Persons, American Geriatrics Society and British Geriatrics Society. Summary of the Updated American Geriatrics Society/British Geriatrics Society clinical practice guideline for prevention of falls in older persons. J Am Geriatr Soc. 2011; 59(1):148-157.

14. Clemson L, Cumming RG, Kendig H, Swann M, Heard R, Taylor K. The effectiveness of a community-based program for reducing the incidence of falls in the elderly: a randomized trial. J Am Geriatr Soc. 2004;52(9):1487-1494.

15. Clemson L, Singh MF, Bundy A, et al. LiFE Pilot Study: A randomised trial of balance and strength training embedded in daily life activity to reduce falls in older adults. Aust Occup Ther J. 2010;57(1): $42-50$.

16. Lamb SE, Becker C, Gillespie LD, et al. Reporting of complex interventions in clinical trials: development of a taxonomy to classify and describe fall-prevention interventions. Trials. 2011;12:125.

17. Folstein MF, Folstein SE, McHugh PR. "Mini-mental state". A practical method for grading the cognitive state of patients for the clinician. J Psychiatr Res. 1975;12(3):189-198.

18. Haran MJ, Cameron ID, Ivers RQ, et al. Effect on falls of providing single lens distance vision glasses to multifocal glasses wearers: VISIBLE randomised controlled trial. BMJ. 2010;340:c2265.

19. Lamb SE, Jorstad-Stein EC, Hauer K, Becker C. Development of a common outcome data set for fall injury prevention trials: the Prevention of Falls Network Europe consensus. J Am Geriatr Soc. Sep 2005;53(9):1618-1622. 
20. Schoenfelder DP. A fall prevention program for elderly individuals. Exercise in long-term care settings. J Gerontol Nurs. Mar 2000;26(3): 43-51.

21. Sakamoto K, Nakamura T, Hagino H, et al. Effects of unipedal standing balance exercise on the prevention of falls and hip fracture among clinically defined high-risk elderly individuals: a randomized controlled trial. Journal Orthop Sci. 2006;11(5):467-472.

22. Faber MJ, Bosscher RJ, Chin APMJ, van Wieringen PC. Effects of exercise programs on falls and mobility in frail and pre-frail older adults: A multicenter randomized controlled trial. Arch Phys Med Rehabil. 2006;87(7):885-896.

23. Ware JE Jr, Sherbourne CD. The MOS 36-item short-form health survey (SF-36). I. Conceptual framework and item selection. Med Care. 1992;30(6):473-483.

24. Herdman M, Gudex C, Lloyd A, et al. Development and preliminary testing of the new five-level version of EQ-5D (EQ-5D-5L). Qual Life Res. 2011;20(10):1727-1736.

25. Guralnik JM, Simonsick EM, Ferrucci L, et al. A short physical performance battery assessing lower extremity function: association with self-reported disability and prediction of mortality and nursing home admission. J Gerontol. 1994;49(2):M85-M94.

26. Hill K, Bernardt J, McGann A, Maltese D, Berkovits D. A new test of dynamic standing balance for stroke patients. Reliability, validity and comparison with healthy elderly. Physiother Canada. 1996;48: 257-262.

27. Peel C, Sawyer Baker P, Roth DL, Brown CJ, Brodner EV, Allman RM. Assessing mobility in older adults: the UAB Study of Aging Life-Space Assessment. Phys Ther. 2005;85(10):1008-1119.

28. Tinetti ME, Ginter SF. The nursing home life-space diameter. A measure of extent and frequency of mobility among nursing home residents. J Am Geriatr Soc. 1990;38(12):1311-1315.

29. Yardley L, Beyer N, Hauer K, Kempen G, Piot-Ziegler C, Todd C. Development and initial validation of the Falls Efficacy ScaleInternational (FES-I). Age Ageing. Nov 2005;34(6):614-619.

30. Hauer K, Yardley L, Beyer N, et al. Validation of the Falls Efficacy Scale and Falls Efficacy Scale International in geriatric patients with and without cognitive impairment: results of self-report and interviewbased questionnaires. Gerontology. 2010;56(2):190-199.
31. Muir SW, Gopaul K, Montero Odasso MM. The role of cognitive impairment in fall risk among older adults: a systematic review and meta-analysis. Age Ageing. 2012;41(3):299-308.

32. Heyn P, Abreu BC, Ottenbacher KJ. The effects of exercise training on elderly persons with cognitive impairment and dementia: a metaanalysis. Arch Phys Med Rehabil. 2004;85(10):1694-1704.

33. Mioshi E, Dawson K, Mitchell J, Arnold R, Hodges JR. The Addenbrooke's Cognitive Examination Revised (ACE-R): a brief cognitive test battery for dementia screening. Int J Geriatr Psychiatry. 2006;21(11):1078-1085.

34. Cameron ID, Murray GR, Gillespie LD, et al. Interventions for preventing falls in older people in nursing care facilities and hospitals. Cochrane Database Syst Rev. 2010(1):CD005465.

35. Liu CJ, Latham NK. Progressive resistance strength training for improving physical function in older adults. Cochrane Database Syst Rev. 2009(3):CD002759.

36. Chodzko-Zajko WJ, Proctor DN, Fiatarone Singh MA, et al. American College of Sports Medicine Position Stand. Exercise and physical activity for older adults. Med Sci Sports Exerc. 2009;41(7):1510-1530.

37. Barker AL, Nitz JC, Low Choy NL, Haines TP. Mobility has a non-linear association with falls risk among people in residential aged care: an observational study. J Physiother. 2012;58(2):117-125.

38. Medicare Benefit Scheme. Available from: http://www.mbsonline.gov. au/. Accessed November 17, 2013.

39. Pharmaceutical Benefit Scheme. Available from: http://www.pbs.gov. $\mathrm{au} / \mathrm{pbs} /$ home AR-DRG costs. Accessed November 17, 2013.

40. Australian Government, The Department of Health. Available from: http://www.health.gov.au/internet/main/publishing.nsf/Content/ Round_14-cost-reports. Accessed on November 17, 2013.

41. Norman R, Church J, Van Den Berg B, Goodall S. Australian HealthRelated Quality of Life Population norms derived from the SF-6D. Aust N Z J Public Health. 37(1):17-23.

42. McCabe C, Brazier J, Gilks $P$, et al. Using rank data to estimate health state utility models. J Health Econ. 2006;25(3):418-431.

43. Elley CR, Kerse N, Chondros P, Robinson E. Intraclass correlation coefficients from three cluster randomized controlled trials in primary and residential health care. Aust $N Z$ J Public Health. $2005 ; 29(5): 461-467$
Clinical Interventions in Aging

\section{Publish your work in this journal}

Clinical Interventions in Aging is an international, peer-reviewed journal focusing on evidence-based reports on the value or lack thereof of treatments intended to prevent or delay the onset of maladaptive correlates of aging in human beings. This journal is indexed on PubMed Central, MedLine, the American Chemical Society's 'Chemical Abstracts

\section{Dovepress}

Service' (CAS), Scopus and the Elsevier Bibliographic databases. The manuscript management system is completely online and includes a very quick and fair peer-review system, which is all easy to use. Visit http://www.dovepress.com/testimonials.php to read real quotes from published authors. 